\title{
The Combined Impact of Chronic Kidney Disease and Diabetes on the Risk of Colorectal Cancer Depends on Sex: A Nationwide Population-Based Study
}

\author{
Hyung Jung $\mathrm{Oh}^{1,2 *}$, Hye Ah Lee ${ }^{3 *}$, Chang Mo Moon ${ }^{4,5}$, and Dong-Ryeol Ryu ${ }^{2,4,5}$ \\ ${ }^{1}$ Ewha Institute of Convergence Medicine and ${ }^{2}$ Research Institute for Human Health Information, \\ Ewha Womans University Mokdong Hospital, Seoul; \\ ${ }^{3}$ Clinical Trial Center, Ewha Womans University Mokdong Hospital, Seoul; \\ ${ }^{4}$ Department of Internal Medicine, College of Medicine, Ewha Womans University, Seoul; \\ ${ }^{5}$ Tissue Injury Defense Research Center, Ewha Womans University, Seoul, Korea.
}

Purpose: Although both chronic kidney disease (CKD) and diabetes mellitus (DM) are considered factors increasing the risk of colorectal cancer (CRC), their impact on CRC is not fully understood. This study was aimed to investigate the impact of CKD, DM, or both diseases on the risk of CRC and to evaluate sex differences therein.

Materials and Methods: Using data from the National Health Insurance Service-Health Examination Cohort in Korea, we conducted a 1:2 matched case-control study. The disease groups consisted of CKD-/DM+ (n=17700), CKD+/DM- (n=22643), and $\mathrm{CKD}+/ \mathrm{DM}+$ groups $(\mathrm{n}=8506)$. After 1:2 matching by age, sex, and health examination year and month, the healthy control group consisted of 97698 individuals.

Results: Multivariate Cox regression analysis showed that the CKD-/DM+, CKD+/DM-, and CKD+/DM+ groups were independently associated with an increased incidence of CRC, compared with controls [hazard ratio (HR), 1.34, 1.31, and 1.63, respectively; all $p<0.001]$. Compared to the controls, adjusted HRs for the cumulative incidence of CRC in the CKD-/DM+, CKD+/DM-, and $\mathrm{CKD}+/ \mathrm{DM}+$ groups were, respectively, 1.32, 1.26, and 1.43 in male and 1.38, 1.39, and 2.00 in female. The HR for CRC incidence was significantly higher for the CKD+/DM+ group than for the CKD-/DM+ or CKD+/DM- group in female; however, this significant difference was not observed in male.

Conclusion: In female, having both CKD and DM significantly increases the risk of CRC, compared with having CKD or DM alone. This study suggests a significant difference in the effect of CKD or DM on the risk of CRC according to sex.

Key Words: Chronic kidney disease, diabetes mellitus, colorectal cancer, incidence, sex

\footnotetext{
Received: March 9, 2020 Revised: April 11, 2020

Accepted: April 20, 2020

Co-corresponding authors: Chang Mo Moon, MD, PhD, Department of Internal Medicine, College of Medicine, Tissue Injury Defense Research Center, Ewha Womans University Mokdong Hospital, 1071 Anyangcheon-ro, Yangcheon-gu, Seoul 07985, Korea. Tel: 82-2-2650-2945, Fax: 82-2-2655-2076, E-mail: drmcm75@naver.com and Dong-Ryeol Ryu, MD, PhD, Department of Internal Medicine, College of Medicine, Tissue Injury Defense Research Center, Ewha Womans University Seoul Hospital, 2-gil 25 Magokdong-ro, Gangseo-gu, Seoul 07804, Korea.

Tel: 82-2-2650-2507, Fax: 82-2-2650-2501, E-mail: drryu@ewha.ac.kr

*Hyung Jung Oh and Hye Ah Lee contributed equally to this work.

-The authors have no potential conflicts of interest to disclose.

(C) Copyright: Yonsei University College of Medicine 2020

This is an Open Access article distributed under the terms of the Creative Commons Attribution Non-Commercial License (https://creativecommons.org/licenses/ by-nc/4.0) which permits unrestricted non-commercial use, distribution, and reproduction in any medium, provided the original work is properly cited.
}

\section{INTRODUCTION}

Worldwide, more than 1 million people develop colorectal cancer (CRC) annually, and the CRC-related mortality rate is almost $33 \%$ in developed countries. ${ }^{1} \mathrm{CRC}$ is the second leading cause of cancer-related death in the US, and it has the second highest crude incidence (54.6) per 100000 population in both male and female in Korea, with an incidence that is still increasing. ${ }^{2,3}$

Research has shown that chronic kidney disease (CKD) is significantly associated with an increased risk of CRC. compared with CRC risk in the general population regardless of comorbidities. ${ }^{4} \mathrm{CKD}$ is prevalent worldwide and has high incidences of $13.1 \%$ in the US and $13.7 \%$ in Korea among individuals 
older than 20 years of age. ${ }^{5,6}$ Patients with CKD may progress to end-stage renal disease and develop subsequent complications. ${ }^{7}$ However, the etiology by which the risk of CRC increases with CKD is still unclear. With respect to the risk of cancer in pre-dialysis CKD, Park, et al. ${ }^{8}$ reported the incidence rate of all neoplasms in pre-dialysis CKD patients, compared with that in matched controls, using Korean nationwide populationbased study: they showed that the incidence of digestive cancer was lower in individuals with pre-dialysis CKD than in matched controls, although they did not provide a reason for the finding.

The leading cause of CKD is diabetes mellitus (DM), which accounts for $44 \%$ of new CKD cases. ${ }^{9}$ Recently, a populationbased, cross-sectional study demonstrated a relative risk of CRC of 2.9 in subjects with a history of type II DM. ${ }^{10}$ Although this phenomenon is similar between male and female, it is more pronounced in individuals with a family history of CRC. ${ }^{11}$ Insulin and its structural homolog insulin-like growth factor-I are believed to promote colorectal carcinogenesis, although issues including any related risk quantification remain unclear. ${ }^{11-14}$ Several studies have reported that CKD and DM each have a significant association with $\mathrm{CRC}$ incidence. ${ }^{4,11,15-17}$ However, to the best our knowledge, no study has compared the relative CRC risk of patients with both CKD and DM with that of healthy control subjects, CKD only patients, or DM only patients. Thus, in the present study, to determine the effect of CKD with or without DM on the risk of CRC, we investigated the association of CKD, DM, or both diseases with the incidence of CRC. Accordingly, we stratified the study population into four groups of healthy control (CKD-/DM-), DM only (CKD-/DM+), CKD only (CKD+/DM-), and CKD with DM (CKD+/DM+) patients, and investigated the relative risk of CRC among the groups. Additionally, sex differences in the risk of CRC were also examined among these four groups.

\section{MATERIALS AND METHODS}

\section{Data source}

This study was conducted using data from the National Health Insurance Service (NHIS)-Health Examination Cohort in the Republic of Korea. Detailed information concerning this cohort is described in Supplementary Material 1 (only online). In this study, the date of the health examination from 2002 to 2003 was considered the index date (it also indicates the baseline period). This study was approved by the Institutional Review Board of the Ewha Womans University Mokdong Hospital (IRB approved number; 2018-01-006).

\section{Study subject cohort}

To evaluate the study cohort for further analyses, we excluded subjects from the baseline population of the NHIS-health examination cohort in accordance with the following criteria: having undergone peritoneal dialysis, hemodialysis, or kidney transplantation $(\mathrm{n}=1733)$ and having inflammatory bowel disease or familial and hereditary polyposis $(n=21056)$. These subjects were identified through claimed records according to diagnosis and/or medical treatment codes. Subjects with a selfreported previous cancer history $(n=2909)$, those diagnosed with any type of cancer $(\mathrm{n}=6739)$, and those who died $(\mathrm{n}=1611)$ within 1 year after the index date were also excluded. Additionally, subjects with missing data in terms of smoking status and body mass index (BMI) were excluded $(n=18751)$. Among the subjects remaining after applying the exclusion criteria $(\mathrm{n}=$ 461996), we formed case and control groups according to the following definitions: prevalent CKD and DM were defined according to claimed records of hospital utilization and drug prescriptions. Prevalent CKD subjects were defined as those who visited an outpatient clinic on at least two different days or those who were hospitalized more than once with a diagnosis code of 'N18, 'N19, 'I12,' 'I13, ‘E10.2,' 'E11.2,' 'E13.2', or 'E14.2' based on the International Classification of Diseases, 10th revision, during the baseline period. Prevalent DM subjects were defined as those with at least two records of prescription insulin or oral hypoglycemic agents with a diagnosis code of 'E10$14{ }^{\prime \prime 18,19}$ The CRC incidence was defined as the first hospitalization with a diagnosis code of 'C18-20' from 1 year after the index date to December 31, 2013.

To achieve the study objectives, prevalent CKD/DM patients were grouped as patients with DM only (CKD-/DM+, $\mathrm{n}=17700)$, with CKD only (CKD+/DM-, n=22643), and with both CKD and $\mathrm{DM}(\mathrm{CKD}+/ \mathrm{DM}+, \mathrm{n}=8506)$. To construct the control group, two control subjects per one patient were selected through individual matching according to sex, age ( \pm 4 years), and health examination month ( \pm 3 months) and year $(n=97698)$. The flow diagram of this study is presented in Supplementary Fig. 1 (only online). A description of the clinical and lifestyle variables is provided in Supplementary Material 2 (only online).

\section{Statistical analysis}

The results are presented as means \pm standard deviations for numeric variables and as numbers of subjects and percentages for categorical variables. As the endpoint, CRC incidence was defined as the first hospitalization with a diagnosis code of 'C18-20' from 1 year after the index date to December 31, 2013. Follow up was calculated from the index date to the date of the first diagnosis of CRC, death, or end of the study (December $31,2013)$. The incidence of CRC was estimated as 100000 person-years. We also used Poisson regression analysis to estimate the incidence rate ratio (IRR) of CRC risk and 95\% confidence intervals (CIs) for each patient group in comparison to controls. The Kaplan-Meier method and log-rank test were used to evaluate the difference in the cumulative incidence of CRC between the control subjects and patients with CKD, DM, or both. The hazard ratios (HRs) of CRC in the disease groups (CKD-/DM+, CKD+/DM-, CKD+/DM+) were estimated using 
the Cox proportional hazards regression model. Its assumption was tested using the Schoenfeld residuals and was satisfied. Using univariate Cox proportional hazards regression analyses, we assessed the individual effects of potential risk factors on CRC risk. Significant $(p<0.1)$ risk factors identified from the univariate analyses [presence of CKD or DM, age, sex, income, BMI, smoking status, alcohol consumption, exercise, Charlson Comorbidity Index (CCI) score, family history of cancer, metformin use, and aspirin or NSAID use] were entered into the Cox proportional hazards regression model. The differences in HR among the patient subgroups according to the presence of $\mathrm{CKD}$, DM, or both were also tested using pairwise comparisons in the multivariate Cox proportional hazards re- gression model. All statistical analyses were conducted using SAS version 9.4 (SAS Institute, Cary, NC, USA), and statistical significance was set as 0.05 in two-sided tests.

\section{RESULTS}

\section{Baseline characteristics of the four groups stratified according to the presence of CKD or DM}

The disease groups consisted of CKD-/DM+ $(n=17700), C K D+/$ DM- $(\mathrm{n}=22643)$, and CKD+/DM+ groups ( $\mathrm{n}=8506)$. After $1: 2$ matching by age, sex, health examination year and month, the healthy control group consisted of 97698 individuals. The base-

Table 1. Baseline Characteristics of the Study Groups Stratified according to the Presence of CKD or Diabetes

\begin{tabular}{|c|c|c|c|c|c|}
\hline & \multirow[b]{2}{*}{$\begin{array}{c}\text { Control } \\
\text { (n=97698) }\end{array}$} & \multirow[b]{2}{*}{$\begin{array}{c}\text { Disease } \\
(\mathrm{n}=48849)\end{array}$} & \multicolumn{3}{|c|}{ Disease group } \\
\hline & & & $\begin{array}{c}\text { CKD-/DM+ } \\
(n=17700)\end{array}$ & $\begin{array}{c}\text { CKD+/DM- } \\
(n=22643)\end{array}$ & $\begin{array}{c}\text { CKD+/DM+ } \\
(n=8506)\end{array}$ \\
\hline Age (yr) & $57.9 \pm 9.7$ & $57.9 \pm 9.7$ & $58.1 \pm 9.4$ & $57.3 \pm 10.0$ & $58.8 \pm 9.2$ \\
\hline Male & $53,172(54.4)$ & 26586 (54.4) & $9913(56.0)$ & 12031 (53.1) & $4,642(54.6)$ \\
\hline \multicolumn{6}{|l|}{ Income* } \\
\hline Medical-aid & $265(0.3)$ & $153(0.3)$ & $48(0.3)$ & $78(0.3)$ & $27(0.3)$ \\
\hline $1 s t-3 r d$ & 23978 (24.5) & 11605 (23.8) & 4379 (24.7) & 5390 (23.8) & 1836 (21.6) \\
\hline 4th-6th & $23127(23.7)$ & 11255 (23.0) & 4237 (23.9) & $5106(22.6)$ & $1912(22.5)$ \\
\hline 7th-8th & 19921 (20.4) & $9931(20.3)$ & 3609 (20.4) & 4531 (20.0) & 1791 (21.1) \\
\hline 9th-10th & 30407 (31.1) & 15905 (32.6) & 5427 (30.7) & 7538 (33.3) & 2940 (34.6) \\
\hline \multicolumn{6}{|l|}{ BMI $\left(\mathrm{kg} / \mathrm{m}^{2}\right)$} \\
\hline$<20$ & $8942(9.2)$ & $2342(4.8)$ & $708(4.0)$ & 1254 (5.5) & $380(4.5)$ \\
\hline $20-24.9$ & $55400(56.7)$ & 24218 (49.6) & $8863(50.1)$ & 11125 (49.1) & $4230(49.7)$ \\
\hline$\geq 25$ & 33356 (34.1) & 22289 (45.6) & $8129(45.9)$ & 10264 (45.3) & 3896 (45.8) \\
\hline \multicolumn{6}{|l|}{ Smoking } \\
\hline Non-smoker & 66974 (68.6) & 33533 (68.6) & 11955 (67.5) & 15647 (69.1) & 5931 (69.7) \\
\hline Ex-smoker & $8363(8.6)$ & 4422 (9.1) & $1626(9.2)$ & $2048(9.0)$ & 748 (8.8) \\
\hline Current smoker & $22361(22.9)$ & 10894 (22.3) & $4119(23.3)$ & 4948 (21.9) & $1827(21.5)$ \\
\hline \multicolumn{6}{|l|}{ Drinking $^{\dagger}$} \\
\hline No-drinking & 58294 (59.9) & 30428 (62.6) & 11095 (63.0) & 13754 (60.9) & $5579(66.0)$ \\
\hline 2-3 times/month & $12927(13.3)$ & $6072(12.5)$ & $2032(11.5)$ & $3015(13.4)$ & $1025(12.1)$ \\
\hline 1-2 times/week & $14102(14.5)$ & $6757(13.9)$ & $2495(14.2)$ & $3211(14.2)$ & $1051(12.4)$ \\
\hline$\geq 3$ times/week & $11974(12.3)$ & $5374(11.1)$ & $1989(11.3)$ & $2587(11.5)$ & $798(9.4)$ \\
\hline \multicolumn{6}{|l|}{ Exercise $^{\ddagger}$} \\
\hline None & $58642(60.9)$ & 27054 (56.3) & $9483(54.4)$ & $13146(59.0)$ & $4425(52.8)$ \\
\hline 1-2 times/week & $19918(20.7)$ & $10345(21.5)$ & $3766(21.6)$ & $4735(21.2)$ & $1844(22.0)$ \\
\hline$\geq 3$ times/week & $17728(18.4)$ & 10692 (22.2) & $4181(24.0)$ & 4403 (19.8) & $2108(25.2)$ \\
\hline CCl score ${ }^{\S}$ & $0.5 \pm 0.8$ & $0.8 \pm 1.0$ & $0.7 \pm 0.9$ & $0.8 \pm 1.1$ & $0.9 \pm 1.1$ \\
\hline Family history of cancer" & $10322(11.9)$ & 4607 (10.6) & $1558(9.9)$ & $2326(11.6)$ & $723(9.6)$ \\
\hline Use of metformin & $92(0.1)$ & $14687(30.1)$ & $9496(53.6)$ & $52(0.2)$ & $5139(60.4)$ \\
\hline Use of aspirin or NSAIDs & $65900(67.5)$ & $37413(76.6)$ & $13636(77.0)$ & 16934 (74.8) & $6843(80.4)$ \\
\hline
\end{tabular}

CKD, chronic kidney disease; DM, diabetes mellitus; BMI, body mass index; CCI, Charlson Comorbidity Index; NSAIDs, non-steroidal anti-inflammatory drugs. Data are expressed as $n(\%)$.

*Income level: the NHIS-health examination cohort provide income level data as medical aid beneficiaries, deciles for insured employees, and deciles for insured self-employed, ${ }^{\dagger}$ Numbers vary due to missing data. (97297, 48631, 17611, 22567, and 8453 subjects for control, disease, CKD-/DM+, CKD+/DM-, and $\mathrm{CKD}+/ \mathrm{DM}+$, respectively), ${ }^{\ddagger}$ Numbers vary due to missing data. (96288, 48091, 17430, 22284, and 8377 subjects for control, disease, CKD-/DM+, CKD+/DM-, and

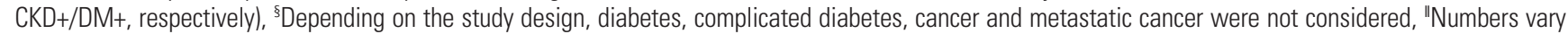
due to missing data $(87032,43382,15753,20121$, and 7508 subjects for control, disease, CKD-/DM+, CKD+/DM-, and CKD+/DM+, respectively). 
line characteristics of the study population are shown in Table 1.

\section{CRC incidence according to CKD or DM}

We initially investigated the difference in CRC incidence according to CKD or DM using the Kaplan-Meier method and log-rank test, which showed that the cumulative incidence of CRC was significantly higher in the CKD or DM group, compared with that in the control group $(p<0.0001)$ (Supplementary Fig. 2, only online). During the median follow-up period of 10.7 years (interquartile range: 10.2-11.2), the incidences of CRC per 100000 person-years were 179.2 (95\% CI, 170.9-187.4) in the control group, 223.3 (95\% CI, 201.3-245.2) in the CKD-/ $\mathrm{DM}+$ group, 234.3 (95\% CI, 214.7-253.9) in the CKD+/DM- group, and 285.4 (95\% CI, 249.6-321.2) in the CKD+/DM+ group. We also estimated IRR using the Poisson model, and the trends were similar to the incidence of CRC (Table 2). These results indicated that the incidence rate of CRC was higher in the disease group than in the control group. When assessed according to sex, similar to the whole population, CRC incidence was higher in the disease group than in the control group for both male and female. However, a difference in the increase in CRC incidence (per 100000 person-years) in the disease groups, compared with the control groups, was observed according to sex: the CRC incidence was $29.8 \%$ higher in the CKD+/DM+ group than in the control group (302.5 vs. 212.5 , respectively) in male and $47.2 \%$ higher in the $\mathrm{CKD}+/ \mathrm{DM}+$ group than in the control group (264.8 vs. 139.8 , respectively) in female.

\section{Impact of CKD or DM on the cumulative incidence of CRC}

To investigate the association between CRC and each vari-

Table 2. Incidence of Colorectal Cancer in the Study Groups according to the Presence of CKD or Diabetes

\begin{tabular}{lrrcc}
\hline \multicolumn{1}{c}{ Group } & Total & $\begin{array}{c}\text { Incident } \\
\text { case }\end{array}$ & $\begin{array}{c}\text { Incidence* } \\
(\mathbf{9 5 \%} \mathbf{C I})\end{array}$ & $\begin{array}{c}\text { IRR } \\
(\mathbf{9 5 \%} \mathbf{C I})\end{array}$ \\
\hline All & & & & \\
\hline Control & 97698 & 1812 & $179.2(170.9-187.4)$ & reference \\
\hline CKD-/DM+ & 17700 & 398 & $223.3(201.3-245.2)$ & $1.2(1.1-1.4)$ \\
\hline CKD+/DM- & 22643 & 549 & $234.3(214.7-253.9)$ & $1.3(1.2-1.4)$ \\
\hline CKD+/DM+ & 8506 & 244 & $285.4(249.6-321.2)$ & $1.6(1.4-1.8)$ \\
\hline Male & & & & \\
\hline Control & 53172 & 1163 & $212.5(200.3-224.7)$ & reference \\
\hline CKD-/DM+ & 9913 & 252 & $254.1(222.7-285.5)$ & $1.2(1.0-1.4)$ \\
\hline CKD+/DM- & 12031 & 344 & $277.4(248.1-306.7)$ & $1.3(1.2-1.5)$ \\
\hline CKD+/DM+ & 4642 & 141 & $302.5(252.6-352.4)$ & $1.4(1.2-1.7)$ \\
\hline Female & & & & \\
\hline Control & 44526 & 649 & $139.8(129.1-150.6)$ & reference \\
\hline CKD-/DM+ & 7787 & 146 & $184.6(154.6-214.5)$ & $1.3(1.1-1.6)$ \\
\hline CKD+/DM- & 10612 & 205 & $185.8(160.4-211.2)$ & $1.3(1.1-1.6)$ \\
\hline CKD+/DM+ & 3864 & 103 & $264.8(213.7-315.9)$ & $1.9(1.5-2.3)$ \\
\hline
\end{tabular}

$\mathrm{Cl}$, confidence interval; $\mathrm{CKD}$, chronic kidney disease; DM, diabetes mellitus; IRR, incidence rate ratio.

*Incidence per 100000 person-years. able, we performed Cox proportional hazards regression analyses. An unadjusted Cox analysis showed that CKD and DM were each significantly associated with an increased incidence of CRC, compared with the controls [HR, 1.26 (95\% CI, 1.131.40) in CKD-/DM+ group; HR, 1.31 (95\% CI, 1.19-1.44) in CKD+/ DM- group]. Furthermore, the HR in the $\mathrm{CKD}+/ \mathrm{DM}+$ group was 1.61 (95\% CI, 1.41-1.84). When all of the variables were adjusted for in the multivariate analysis, CKD, DM, and CKD with DM were each still independently correlated with an increased incidence of CRC, compared with the controls [HR, 1.34 (95\% CI, 1.16-1.55) in CKD-/DM+ group; HR, 1.31 (95\% CI, 1.18-1.46) in CKD+/DM- group; and HR, 1.63 (95\% CI, 1.371.94) in CKD+ /DM+ group] (Table 3). In the multivariate Cox proportional hazards regression model, older age (HR, 1.06; 95\% CI, 1.06-1.07), male (HR, 1.55; 95\% CI, 1.41-1.72), current smoker status (HR, 1.17; 95\% CI, 1.06-1.30), frequent alcohol drinking ( $\geq 3$ times/week) (HR, 1.36; 95\% CI, 1.20-1.53), and an increased CCI score (HR, 1.09; 95\% CI, 1.04-1.13) were significantly associated with the risk of CRC, whereas the incidence of $\mathrm{CRC}$ was significantly decreased in those with a higher income, compared with a lower income (HR, 0.98; 95\% CI, 0.97-1.00) (Table 3).

\section{Difference in the impact of CKD, DM, or both on CRC} incidence between male and female

To examine whether the impact of CKD, DM, or both on CRC incidence differed between male and female, we performed the Kaplan-Meier method with log-rank test according to sex. Consequently, the cumulative incidence of CRC was significantly increased in the CKD-/DM+, CKD+/DM- and CKD+/ $\mathrm{DM}+$ groups, compared with the control group, in both male and female. However, in male, no significant difference in the cumulative incidence of CRC was observed between the CKD/DM+ and CKD+/DM+ groups or between the CKD+/DM- and $\mathrm{CKD}+/ \mathrm{DM}+$ groups (Fig. 1A). On the other hand, in female, the cumulative incidence of CRC was significantly higher in the $\mathrm{CKD}+/ \mathrm{DM}+$ group than in the $\mathrm{CKD}-/ \mathrm{DM}+$ or $\mathrm{CKD}+/ \mathrm{DM}$ group (CKD-/DM+ vs. CKD+/DM+, $p=0.005$, and CKD+/DMvs. $\mathrm{CKD}+/ \mathrm{DM}+, p=0.003$ ) (Fig. 1B). According to multivariate Cox regression analysis adjusting for all possible variables, CKD, DM, or both was independently associated with an increase in CRC incidence compared, with the controls, in both male and female (Table 4). In this model, the adjusted HRs in the $\mathrm{CKD}-/ \mathrm{DM}+, \mathrm{CKD}+/ \mathrm{DM}-$, and $\mathrm{CKD}+/ \mathrm{DM}+$ groups were, respectively, $1.32(p=0.002), 1.26(p<0.001)$, and $1.43(p=0.002)$ in male, and $1.38(p=0.010), 1.39(p<0.001)$, and $2.00(p<0.001)$ in female.

The other independent risk factors associated with the risk of CRC in male (older age, economic income, current smoker status, frequent alcohol drinking, and higher CCI score) were consistent with those in the whole population (Table 4). In contrast, in female, economic income and frequent alcohol drinking were not significantly associated with the risk of CRC; how- 
Table 3. Cox Proportional Hazards Regression Analysis of the Cumulative Incidence of Colorectal Cancer

\begin{tabular}{|c|c|c|c|c|}
\hline & \multicolumn{2}{|c|}{ Unadjusted Cox regression analysis } & \multicolumn{2}{|c|}{ Multivariate Cox regression analysis ${ }^{*}$} \\
\hline & Crude HR ( $95 \%$ CI) & $p$ value & Adjusted HR (95\% CI) & $p$ value \\
\hline \multicolumn{5}{|l|}{ Group (vs. control) } \\
\hline CKD-/DM+ & $1.26(1.13-1.40)$ & $<0.001$ & $1.34(1.16-1.55)$ & $<0.001$ \\
\hline CKD+/DM- & $1.31(1.19-1.44)$ & $<0.001$ & $1.31(1.18-1.46)$ & $<0.001$ \\
\hline CKD+/DM+ & $1.61(1.41-1.84)$ & $<0.001$ & $1.63(1.37-1.94)$ & $<0.001$ \\
\hline Age (per 1-year increase) & $1.06(1.06-1.06)$ & $<0.001$ & $1.06(1.06-1.07)$ & $<0.001$ \\
\hline Male (vs. female) & $1.46(1.36-1.57)$ & $<0.001$ & $1.55(1.41-1.72)$ & $<0.001$ \\
\hline Income (continuous variable from Medical-aid to 10th) & $0.97(0.96-0.99)$ & $<0.001$ & $0.98(0.97-1.00)$ & 0.014 \\
\hline \multicolumn{5}{|l|}{ BMI (vs. 20-25 kg/m²) } \\
\hline$<20$ & $1.35(1.19-1.53)$ & $<0.001$ & $1.13(0.98-1.29)$ & 0.094 \\
\hline$\geq 25$ & $0.96(0.89-1.04)$ & 0.337 & $1.03(0.94-1.11)$ & 0.557 \\
\hline \multicolumn{5}{|l|}{ Smoking (vs. non-smoker) } \\
\hline Ex-smoker & $1.23(1.09-1.39)$ & $<0.001$ & $1.05(0.91-1.21)$ & 0.499 \\
\hline Current smoker & $1.25(1.15-1.36)$ & $<0.001$ & $1.17(1.06-1.30)$ & 0.003 \\
\hline \multicolumn{5}{|l|}{ Drinking (vs. non-drinker) } \\
\hline 2-3 times/month & $1.00(0.89-1.12)$ & 0.974 & $1.13(0.99-1.28)$ & 0.063 \\
\hline 1-2 times/week & $1.05(0.94-1.17)$ & 0.393 & $1.15(1.01-1.30)$ & 0.030 \\
\hline$\geq 3$ times/week & $1.53(1.38-1.69)$ & $<0.001$ & $1.36(1.20-1.53)$ & $<0.001$ \\
\hline \multicolumn{5}{|l|}{ Exercise (vs. $\geq 3$ times/week) } \\
\hline None & $1.05(0.95-1.15)$ & 0.346 & $1.03(0.93-1.14)$ & 0.584 \\
\hline 1-2 times/week & $0.88(0.78-0.99)$ & 0.031 & $0.99(0.88-1.13)$ & 0.925 \\
\hline CCI score (per 1 unit increase) & $1.21(1.16-1.25)$ & $<0.001$ & $1.09(1.04-1.13)$ & $<0.001$ \\
\hline Family history of cancer (vs. no history) & $0.84(0.74-0.95)$ & 0.006 & $1.03(0.90-1.17)$ & 0.670 \\
\hline Use of metformin (vs. non-use) & $1.19(1.07-1.34)$ & 0.002 & $0.92(0.78-1.08)$ & 0.302 \\
\hline Use of aspirin or NSAIDs (vs. non-use) & $1.13(1.04-1.22)$ & 0.004 & $0.99(0.90-1.08)$ & 0.750 \\
\hline
\end{tabular}

HR, hazard ratio; Cl, confidence interval; CKD, chronic kidney disease; DM, diabetes mellitus; BMI, body mass index; CCI, Charlson Comorbidity Index; NSAIDs, nonsteroidal anti-inflammatory drugs.

${ }^{*}$ Adjusted for presence of CKD or DM, age, sex, income, BMI, smoker habits, alcohol intake, exercise, CCI score, family history of cancer, metformin use, use of aspirin or NSAIDs.

A

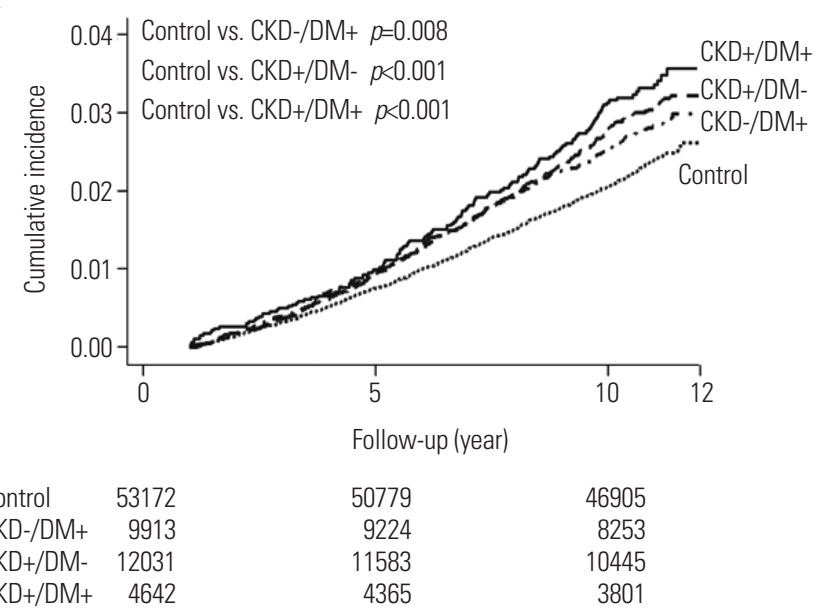

B

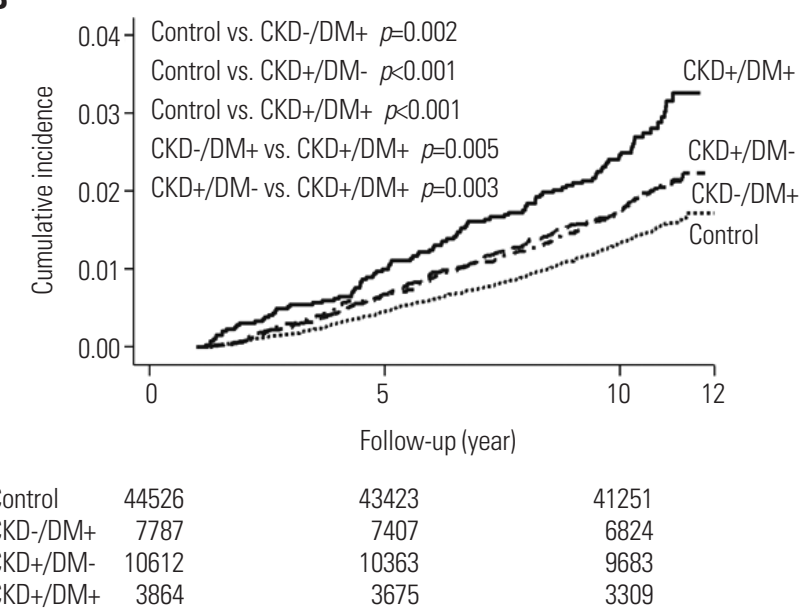

Fig. 1. Kaplan-Meier curves for CRC incidence according to the presence of CKD or DM in male (A) and female (B). The cumulative incidence of CRC was significantly increased in the CKD-/DM+, CKD+/DM-, and CKD+/DM+ groups, compared with the control groups, in both male and female. However, in male, no significant difference was observed in the cumulative incidence of CRC between the CKD+/DM+ and the CKD-/DM+ or CKD+/DM- groups (A). In contrast, the cumulative incidence of CRC was significantly increased in the CKD+/DM+ group, compared with the CKD-/DM+ or CKD+/DM- group, in female (B). CRC, colorectal cancer; CKD, chronic kidney disease; DM, diabetes mellitus. 
Hyung Jung Oh, et al.

Table 4. Cox Proportional Hazards Regression Analysis of the Cumulative Incidence of Colorectal Cancer according to Sex

\begin{tabular}{|c|c|c|c|c|c|c|c|c|}
\hline & \multicolumn{4}{|c|}{ Male } & \multicolumn{4}{|c|}{ Female } \\
\hline & \multicolumn{2}{|c|}{$\begin{array}{c}\text { Unadjusted } \\
\text { Cox regression analysis }\end{array}$} & \multicolumn{2}{|c|}{$\begin{array}{c}\text { Multivariate } \\
\text { Cox regression analysis }\end{array}$} & \multicolumn{2}{|c|}{$\begin{array}{c}\text { Unadjusted } \\
\text { Cox regression analysis }\end{array}$} & \multicolumn{2}{|c|}{$\begin{array}{c}\text { Multivariate } \\
\text { Cox regression analysis }{ }^{*}\end{array}$} \\
\hline & $\begin{array}{l}\text { Crude HR } \\
\text { (95\% CI) }\end{array}$ & $p$ value & $\begin{array}{l}\text { Adjusted HR } \\
\text { (95\% Cl) }\end{array}$ & $p$ value & $\begin{array}{l}\text { Crude HR } \\
\text { (95\% CI) }\end{array}$ & $p$ value & $\begin{array}{l}\text { Adjusted HR } \\
\text { (95\% CI) }\end{array}$ & $p$ value \\
\hline \multicolumn{9}{|l|}{ Group (vs. control) } \\
\hline CKD-/DM+ & $1.20(1.05-1.38)$ & 0.008 & $1.32(1.11-1.59)$ & 0.002 & $1.33(1.11-1.59)$ & 0.002 & $1.38(1.08-1.75)$ & 0.010 \\
\hline CKD+/DM- & $1.31(1.16-1.47)$ & $<0.001$ & $1.26(1.11-1.44)$ & $<0.001$ & $1.33(1.14-1.56)$ & $<0.001$ & $1.39(1.18-1.65)$ & $<0.001$ \\
\hline CKD+/DM+ & $1.44(1.21-1.71)$ & $<0.001$ & $1.43(1.14-1.79)$ & 0.002 & $1.92(1.56-2.36)$ & $<0.001$ & $2.00(1.52-2.63)$ & $<0.001$ \\
\hline Age (per 1-year increase) & $1.07(1.06-1.07)$ & $<0.001$ & $1.07(1.06-1.07)$ & $<0.001$ & $1.06(1.05-1.07)$ & $<0.001$ & $1.06(1.05-1.07)$ & $<0.001$ \\
\hline $\begin{array}{l}\text { Income (continuous variable from } \\
\text { Medical-aid to 10th) }\end{array}$ & $0.96(0.95-0.97)$ & $<0.001$ & $0.98(0.97-1.00)$ & 0.040 & $0.98(0.96-1.00)$ & 0.061 & $0.99(0.97-1.01)$ & 0.259 \\
\hline \multicolumn{9}{|l|}{ BMI (vs. $20-25$ kg/m²) } \\
\hline$<20$ & $1.41(1.21-1.64)$ & $<0.001$ & $1.15(0.97-1.36)$ & 0.103 & $1.24(0.99-1.54)$ & 0.061 & $1.07(0.84-1.37)$ & 0.581 \\
\hline$\geq 25$ & $0.91(0.82-1.00)$ & 0.049 & $1.02(0.92-1.13)$ & 0.740 & $1.10(0.97-1.24)$ & 0.141 & $1.03(0.91-1.18)$ & 0.628 \\
\hline \multicolumn{9}{|l|}{ Smoking (vs. non-smoker) } \\
\hline Ex-smoker & $0.95(0.83-1.09)$ & 0.467 & $1.03(0.89-1.19)$ & 0.735 & $1.56(0.95-2.55)$ & 0.080 & $1.39(0.81-2.36)$ & 0.231 \\
\hline Current smoker & $0.96(0.87-1.06)$ & 0.386 & $1.16(1.04-1.29)$ & 0.010 & $1.74(1.34-2.27)$ & $<0.001$ & $1.39(1.04-1.86)$ & 0.028 \\
\hline \multicolumn{9}{|l|}{ Drinking (vs. non-drinker) } \\
\hline 2-3 times/month & $0.83(0.73-0.96)$ & 0.010 & $1.09(0.94-1.27)$ & 0.254 & $0.99(0.80-1.23)$ & 0.952 & $1.23(0.98-1.55)$ & 0.079 \\
\hline 1-2 times/week & $0.86(0.76-0.97)$ & 0.014 & $1.15(1.01-1.32)$ & 0.041 & $0.90(0.64-1.24)$ & 0.507 & $1.09(0.76-1.56)$ & 0.653 \\
\hline$\geq 3$ times/week & $1.24(1.11-1.39)$ & $<0.001$ & $1.37(1.20-1.55)$ & $<0.001$ & $0.98(0.61-1.59)$ & 0.943 & $1.11(0.67-1.83)$ & 0.683 \\
\hline \multicolumn{9}{|l|}{ Exercise (vs. $\geq 3$ times/week) } \\
\hline None & $1.01(0.90-1.13)$ & 0.843 & $0.94(0.83-1.06)$ & 0.275 & $1.37(1.15-1.63)$ & $<0.001$ & $1.27(1.05-1.54)$ & 0.013 \\
\hline 1-2 times/week & $0.79(0.69-0.91)$ & $<0.001$ & $0.97(0.84-1.12)$ & 0.660 & $1.06(0.83-1.34)$ & 0.651 & $1.07(0.83-1.39)$ & 0.596 \\
\hline CCI score (per 1 unit increase) & $1.25(1.20-1.31)$ & $<0.001$ & $1.09(1.03-1.15)$ & 0.003 & $1.22(1.15-1.28)$ & $<0.001$ & $1.08(1.01-1.15)$ & 0.019 \\
\hline Family history of cancer (vs. no history) & $0.83(0.71-0.98)$ & 0.027 & $1.02(0.87-1.21)$ & 0.780 & $0.85(0.69-1.04)$ & 0.114 & $1.04(0.84-1.28)$ & 0.709 \\
\hline Use of metformin (vs. non-use) & $1.16(1.01-1.34)$ & 0.040 & $0.92(0.75-1.14)$ & 0.442 & $1.26(1.05-1.51)$ & 0.013 & $0.91(0.70-1.19)$ & 0.478 \\
\hline Use of aspirin or NSAIDs (vs. non-use) & $1.19(1.08-1.31)$ & $<0.001$ & $0.97(0.87-1.08)$ & 0.610 & $1.27(1.09-1.48)$ & 0.003 & $1.02(0.86-1.21)$ & 0.839 \\
\hline
\end{tabular}

$\mathrm{HR}$, hazard ratio; $\mathrm{Cl}$, confidence interval; CKD, chronic kidney disease; DM, diabetes mellitus; BMI, body mass index; CCI, Charlson Comorbidity Index; NSAIDs, nonsteroidal anti-inflammatory drugs.

${ }^{*}$ Adjusted for presence of CKD or DM, age, sex, income, BMI, smoker habits, alcohol intake, exercise, CCI score, family history of cancer, metformin use, use of aspirin or NSAIDs.

ever, a lack of exercise (vs. $\geq 3$ times/week) was independently associated with the risk of CRC (HR, 1.27; $p=0.013$ ) (Table 4).

To evaluate the differential impact of CKD, DM, or both on CRC incidence in male and female, adjusted HRs were compared among the disease groups. The HR for CRC incidence was significantly higher in the $\mathrm{CKD}+/ \mathrm{DM}+$ group than in the $\mathrm{CKD}$-/DM+ or CKD+/DM- groups in the whole population ( $p=$ 0.027 and $p=0.025$, respectively) and in female ( $p=0.007$ and $p=0.018$, respectively) after adjusting for all possible variables. However, no statistical difference was observed in the cumulative incidence of CRC between the CKD+/DM+ and CKD-/ $\mathrm{DM}+$ or CKD+/DM- groups in male (Fig. 2).

\section{DISCUSSION}

The present study indicated that individuals with CKD or DM face a significantly increased risk of CRC, compared with non- diabetic and non-CKD populations, irrespective of sex. However, in female, CRC incidence was significantly higher in $\mathrm{CKD}+/ \mathrm{DM}+$ individuals than in CKD-/DM+ or CKD+/DM- individuals, which was not observed in male. In addition, the clinical and environmental risk factors associated with the risk of CRC differed between male and female.

Consistent with several previous studies, ${ }^{4,11,15-17}$ CKD and DM individually were shown to be positively associated with the incidence of CRC in our study. The increased prevalence of CKD is likely attributable to a progressively aging population and increased prevalences of obesity, diabetes, and hypertension. ${ }^{20}$ The association between CRC and DM was first suggested in 1932 and has since been confirmed unequivocally in several observational studies. ${ }^{14-16,21-26} \mathrm{~A}$ recent meta-analysis reported a relationship between diabetes and increased risk of CRC in both female and male. ${ }^{23} \mathrm{~A}$ causal association between DM and CRC is biologically reasonable. DM is characterized by high insulin levels that can stimulate cell prolifera- 


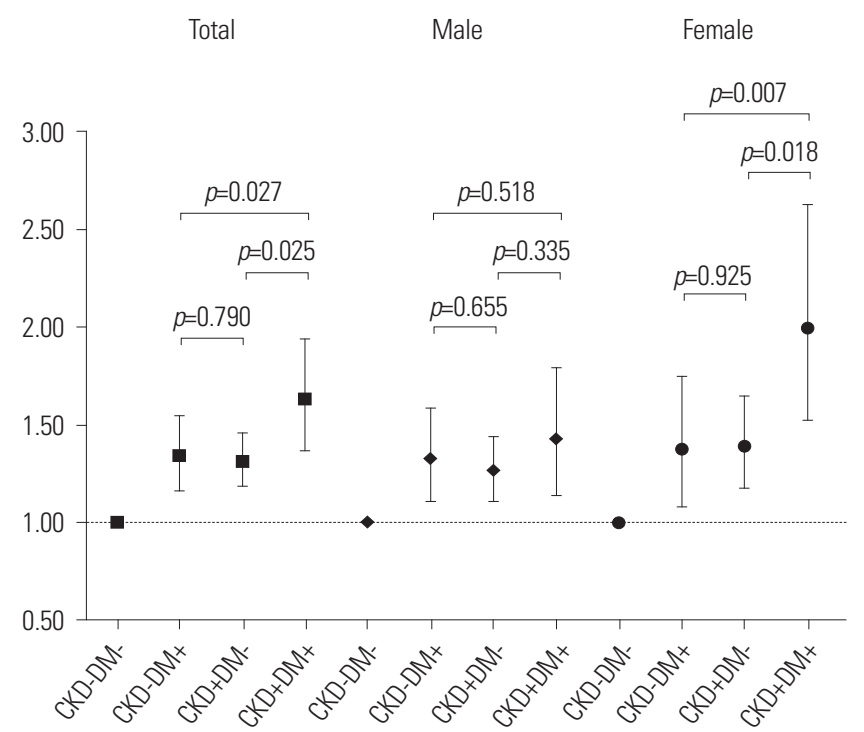

Fig. 2. Differential impact of CKD, DM, or both on CRC incidence between male and female. The HR for CRC incidence was significantly higher in the $C K D+/ D M+$ group, compared with the CKD-/DM+ or CKD+/DM- group, in the whole population ( $p=0.027$ and $p=0.025$, respectively) and in female $(p=0.007$ and $p=0.018$, respectively) after adjusting for all possible variables (age, sex, income, BMI, smoker habit, alcohol consumption, exercise, $\mathrm{CCl}$ score, family history of cancer, metformin use, and aspirin or NSAID use). However, no statistical difference was observed in the cumulative incidence of CRC between the CKD+/DM+ group and CKD-/DM+ or CKD+/DM- group in male. CKD, chronic kidney disease; DM, diabetes mellitus; CRC, colorectal cancer; HR, hazard ratio.

tion via two pathways: a minor and major pathway. These pathways may result in hypersecretion of insulin-like growth factor, ${ }^{11}$ which plays a pivotal role in colorectal carcinogenesis. ${ }^{11,27}$ Other factors associated with insulin resistance, such as high levels of triglycerides or glucose, have been linked to colorectal carcinogenesis. ${ }^{28}$ We also found that both DM and CKD were independent risk factors for CRC in our study. CKD was significantly associated with an increased incidence of CRC irrespective of DM. Moreover, the incidence rate of CRC was higher in the CKD+/DM- group than in the CKD-/DM+ group, although the difference was not statistically significant. To date, the risk of CRC in pre-dialytic CKD patients has been examined in a few studies, including a recent study by $\mathrm{Wu}$, et al. ${ }^{4,29}$ Compared with prior studies, the median follow-up period was longer and more study subjects were examined in our study. To investigate the effect of CKD or DM on increased incidence of CRC, we analyzed the data after stratifying the patients according to the presence of CKD or DM (CKD-/DM+, $\mathrm{CKD}+/ \mathrm{DM}-$, and $\mathrm{CKD}+/ \mathrm{DM}+$ ).

Interestingly, multivariate Cox analysis showed that, in female, CRC incidence was significantly higher in the $\mathrm{CKD}+$ / $\mathrm{DM}+$ group than in the $\mathrm{CKD}-/ \mathrm{DM}+$ or $\mathrm{CKD}+/ \mathrm{DM}$ - group. However, in male, the CRC incidence in the CKD+/DM+ group was not different from that in the CKD-/DM+ or CKD+/DM- group. This result suggests a significant sex difference in the effects of CKD and DM on the risk of CRC. This phenomenon can be explained by several reasons. First, the effects of other clinical or environmental factors on the risk of CRC differ between male and female. The incidence of CRC was higher in the male than in the female control subjects (212.5 per 100000 person-years vs. 139.8 per 100000 personyears, respectively), indicating that other factors besides CKD and DM may have a greater impact on CRC risk in male than in female. However, the causes of these results are difficult to completely explain, and further studies are needed.

Our results highlighted sex differences for other clinical and environmental factors associated with the risk of CRC. Lower economic income and frequent alcohol drinking were significant risk factors for CRC in male, but not in female, while the lack of exercise was a risk factor for CRC in female, but not in male. In traditional Korean society and in accordance with Confucian principles, drinking is acceptable for male but not female. ${ }^{30}$ Although contemporary Korean female drink at a younger age and consume more alcohol, compared with prior generations, the observed sex difference in the rate of alcohol drinking, with higher consumption by male than female, is greater in Korea than in Australia and the US. ${ }^{31}$ In addition, Fedirko, et al. ${ }^{32}$ showed a stronger relative risk for moderate drinkers, compared with non-/occasional drinkers, for male than female. In contrast, regular physical activity, either occupational or leisure time, has been shown to be associated with protection from CRC. ${ }^{33}$ However, according to Kim, et al..${ }^{34}$ the physical activity status, perceived self-efficacy, and benefits of physical activity were significantly lower in Korean female than in Korean male. Thus, frequent alcohol drinking in male and less physical activity in female could explain the higher risk of CRC in this study.

There were several limitations to our study. First, the NHISHealth Examination Cohort was established based on administrative data for health insurance claims instead of clinical data for disease progress. Therefore, we could not analyze the data according to CKD stage classified based on laboratory results. However, CKD was defined according to the same method used in previously published papers. ${ }^{4,35-38}$ Second, study subjects were selected by matching age and sex, as susceptibility to chronic diseases increases with age. Nevertheless, due to a failure to take into account new cases of CKD or DM onset during the observation period, the association may be diluted in terms of misclassification bias. Third, this study was a retrospective design, and thus, selection bias could not be avoided completely. Fourth, we included all diabetic patients (type I and II DM) in this study. Most studies of the association between DM and CRC investigated patients with type II DM..$^{21,23,39}$ However, type II DM is significantly more prevalent than type I DM in Korea. The prevalence of type I DM is approximately $0.017 \%$, whereas the prevalence of type II DM is $8.0 \%$ in Korea. ${ }^{18,40}$ Finally, since this study included only Korean individuals, our findings should be interpreted with caution when attempting to apply them to other ethnicities. 
In conclusion, both DM and CKD alone, as well as CKD plus $\mathrm{DM}$, were independently associated with an increased incidence of CRC, compared with healthy controls. CRC incidence was significantly increased in female with both CKD and DM compared with those with CKD only or DM only; however, this phenomenon was not observed in male. Our results suggest a significant difference in the effect of CKD or DM on the risk of CRC according to sex. However, prospective studies including various ethnic populations are needed to verify our results.

\section{ACKNOWLEDGEMENTS}

This work was supported by the Ewha Womans University Research Grant of 2020.

This work was supported by a National Research Foundation of Korea (NRF) grant funded by the Korea government (MSIT) (grant number 2010-0027945, Chang Mo Moon and Dong-Ryeol Ryu). This research was also supported by the Basic Science Research Program through the National Research Foundation of Korea (NRF), funded by the Ministry of Education (grant number NRF-2017R1D1A1B03035311, Chang Mo Moon) and by Science, ICT, \& Future Planning (grant number NRF-2017R1D1A1A02017503, Hyung Jung Oh).

\section{AUTHOR CONTRIBUTIONS}

Conceptualization: Hyung Jung Oh and Chang Mo Moon. Data curation: all authors. Formal analysis: Hye Ah Lee. Funding acquisition: all authors. Investigation: Hyung Jung Oh, Hye Ah Lee, and Chang Mo Moon. Methodology: Hyung Jung Oh and Hye Ah Lee. Project administration: Chang Mo Moon and Dong-Ryeol Ryu. Resources: Chang Mo Moon and Dong-Ryeol Ryu. Software: Hyung Jung Oh and Hye Ah Lee. Supervision: Chang Mo Moon and Dong-Ryeol Ryu. Validation: Chang Mo Moon and Dong-Ryeol Ryu. Visualization: Hyung Jung Oh and Hye Ah Lee. Writing_-original draft: Hyung Jung Oh, Hye Ah Lee, and Chang Mo Moon. Writing_-review \& editing: Chang Mo Moon and Dong-Ryeol Ryu. Approval of final manuscript: all authors.

\section{ORCID iDs}

Hyung Jung Oh

Hye Ah Lee

https://orcid.org/0000-0002-4281-696X

Chang Mo Moon https://orcid.org/0000-0003-2550-913X

Dong-Ryeol Ryu https://orcid.org/0000-0002-5309-7606

\section{REFERENCES}

1. Siegel R, Ward E, Brawley O, Jemal A. Cancer statistics, 2011: the impact of eliminating socioeconomic and racial disparities on premature cancer deaths. CA Cancer J Clin 2011;61:212-36.

2. Oh CM, Won YJ, Jung KW, Kong HJ, Cho H, Lee JK, et al. Cancer Statistics in Korea: incidence, mortality, survival, and prevalence in 2013. Cancer Res Treat 2016;48:436-50.

3. Shin A, Jung KW, Woo H, Jeong SY. Colorectal cancer incidence in Korea is not the highest in the world. Cancer Res Treat 2016;48: 864-7.

4. Wu MY, Chang TC, Chao TY, Huang MT, Lin HW. Risk of colorec- tal cancer in chronic kidney disease: a matched cohort study based on administrative data. Ann Surg Oncol 2013;20:3885-91.

5. Levey AS, Coresh J. Chronic kidney disease. Lancet 2012;379:16580.

6. Kim S, Lim CS, Han DC, Kim GS, Chin HJ, Kim SJ, et al. The prevalence of chronic kidney disease (CKD) and the associated factors to CKD in urban Korea: a population-based cross-sectional epidemiologic study. J Korean Med Sci 2009;24 Suppl:S11-21.

7. Zhang L, Wang F, Wang L, Wang W, Liu B, Liu J, et al. Prevalence of chronic kidney disease in China: a cross-sectional survey. Lancet 2012;379:815-22.

8. Park S, Lee S, Kim Y, Lee Y, Kang MW, Han K, et al. Risk of cancer in pre-dialysis chronic kidney disease: a nationwide populationbased study with a matched control group. Kidney Res Clin Pract 2019;38:60-70.

9. Centers for Disease Control and Prevention (CDC). Incidence of end-stage renal disease among persons with diabetes--United States, 1990-2002. MMWR Morb Mortal Wkly Rep 2005;54:1097100.

10. Sandhu MS, Luben R, Khaw KT. Self reported non-insulin dependent diabetes, family history, and risk of prevalent colorectal cancer: population based, cross sectional study. J Epidemiol Community Health 2001;55:804-5.

11. Giovannucci E. Insulin, insulin-like growth factors and colon cancer: a review of the evidence. J Nutr 2001;131:3109S-20S.

12. Potter JD. Colorectal cancer: molecules and populations. J Natl Cancer Inst 1999;91:916-32.

13. Reaven GM. Insulin resistance: a chicken that has come to roost. Ann N Y Acad Sci 1999;892:45-57.

14. Schoen RE, Tangen CM, Kuller LH, Burke GL, Cushman M, Tracy RP, et al. Increased blood glucose and insulin, body size, and incident colorectal cancer. J Natl Cancer Inst 1999;91:1147-54.

15. Jee SH, Ohrr H, Sull JW, Yun JE, Ji M, Samet JM. Fasting serum glucose level and cancer risk in Korean men and women. JAMA 2005; 293:194-202.

16. Nilsen TI, Vatten LJ. Prospective study of colorectal cancer risk and physical activity, diabetes, blood glucose and BMI: exploring the hyperinsulinaemia hypothesis. Br J Cancer 2001;84:417-22.

17. Kiberd B. Colorectal cancer screening in kidney disease patients: working backwards. Nephrol Dial Transplant 2013;28:774-7.

18. Song SO, Song YD, Nam JY, Park KH, Yoon JH, Son KM, et al. Epidemiology of type 1 diabetes mellitus in Korea through an investigation of the national registration project of type 1 diabetes for the reimbursement of glucometer strips with additional analyses using claims data. Diabetes Metab J 2016;40:35-45.

19. National Evidence-Based Healthcare Collaborating Agency. A Comparative Study on the Uses of Statin and the Risk of Diabetes Mellitus. Seoul: National Evidence-Based Healthcare Collaborating Agency; 2015.

20. Coresh J, Selvin E, Stevens LA, Manzi J, Kusek JW, Eggers P, et al. Prevalence of chronic kidney disease in the United States. JAMA 2007;298:2038-47.

21. Hu FB, Manson JE, Liu S, Hunter D, Colditz GA, Michels KB, et al. Prospective study of adult onset diabetes mellitus (type 2) and risk of colorectal cancer in women. J Natl Cancer Inst 1999;91:542-7.

22. Khaw KT, Wareham N, Bingham S, Luben R, Welch A, Day N. Preliminary communication: glycated hemoglobin, diabetes, and incident colorectal cancer in men and women: a prospective analysis from the European prospective investigation into cancer-Norfolk study. Cancer Epidemiol Biomarkers Prev 2004;13:915-9.

23. Larsson SC, Giovannucci E, Wolk A. Diabetes and colorectal cancer incidence in the cohort of Swedish men. Diabetes Care 2005;28: 1805-7. 
24. Limburg PJ, Anderson KE, Johnson TW, Jacobs DR Jr, Lazovich D, Hong CP, et al. Diabetes mellitus and subsite-specific colorectal cancer risks in the Iowa Women's Health Study. Cancer Epidemiol Biomarkers Prev 2005;14:133-7.

25. Steenland K, Nowlin S, Palu S. Cancer incidence in the National Health and Nutrition Survey I. Follow-up data: diabetes, cholesterol, pulse and physical activity. Cancer Epidemiol Biomarkers Prev 1995;4:807-11.

26. Will JC, Galuska DA, Vinicor F, Calle EE. Colorectal cancer: another complication of diabetes mellitus? Am J Epidemiol 1998;147:81625.

27. Sandhu MS, Dunger DB, Giovannucci EL. Insulin, insulin-like growth factor-I (IGF-I), IGF binding proteins, their biologic interactions, and colorectal cancer. J Natl Cancer Inst 2002;94:972-80.

28. McKeown-Eyssen G. Epidemiology of colorectal cancer revisited: are serum triglycerides and/or plasma glucose associated with risk? Cancer Epidemiol Biomarkers Prev 1994;3:687-95.

29. Vajdic CM, McDonald SP, McCredie MR, van Leeuwen MT, Stewart $\mathrm{JH}$, Law M, et al. Cancer incidence before and after kidney transplantation. JAMA 2006;296:2823-31.

30. Kim W, Kim S. Women's alcohol use and alcoholism in Korea. Subst Use Misuse 2008;43:1078-87.

31. French DJ, Sargent-Cox KA, Kim S, Anstey KJ. Gender differences in alcohol consumption among middle-aged and older adults in Australia, the United States and Korea. Aust N Z J Public Health 2014; 38:332-9.

32. Fedirko V, Tramacere I, Bagnardi V, Rota M, Scotti L, Islami F, et al.
Alcohol drinking and colorectal cancer risk: an overall and doseresponse meta-analysis of published studies. Ann Oncol 2011;22: 1958-72.

33. Wolin KY, Yan Y, Colditz GA, Lee IM. Physical activity and colon cancer prevention: a meta-analysis. Br J Cancer 2009;100:611-6.

34. Kim HK, Kim MJ, Park CG, Kim HO. Gender differences in physical activity and its determinants in rural adults in Korea. J Clin Nurs 2010;19:876-83.

35. Quan H, Sundararajan V, Halfon P, Fong A, Burnand B, Luthi JC, et al. Coding algorithms for defining comorbidities in ICD-9-CM and ICD-10 administrative data. Med Care 2005;43:1130-9.

36. Kwon YE, Choi HY, Kim S, Ryu DR, Oh HJ; ESRD Registry Committee of the Korean Society of Nephrology. Fracture risk in chronic kidney disease: a Korean population-based cohort study. Kidney Res Clin Pract 2019;38:220-8.

37. Kim KM, Oh HJ, Choi HY, Lee H, Ryu DR. Impact of chronic kidney disease on mortality: a nationwide cohort study. Kidney Res Clin Pract 2019;38:382-90.

38. Oh HJ, Lee HA, Moon CM, Ryu DR. Incidence risk of various types of digestive cancers in patients with pre-dialytic chronic kidney disease: a nationwide population-based cohort study. PLoS One 2018; 13:e0207756.

39. Yang YX, Hennessy S, Lewis JD. Insulin therapy and colorectal cancer risk among type 2 diabetes mellitus patients. Gastroenterology 2004;127:1044-50.

40. Noh J. The diabetes epidemic in Korea. Endocrinol Metab (Seoul) 2016;31:349-53 